Integrating Concepts of Material Mechanics, Ligand Chemistry, Dimensionality and Degradation to Control Differentiation of

\title{
Mesenchymal Stem Cells
}

Matthew G. Haugha,b and Sarah C. Heilshorn ${ }^{\text {a* }}$

${ }^{a}$ Department of Materials Science and Engineering, Stanford University, Stanford, CA, USA

${ }^{\mathrm{b}}$ Tissue Engineering Research Group, Department of Anatomy, Royal College of

Surgeons in Ireland, 123 St. Stephens Green, Dublin 2, Dublin, Ireland.

* Corresponding Author

Prof. S. Heilshorn

476 Lomita Mall

McCullough Rm 246

Stanford, CA

94305, USA

heilshorn@stanford.edu

Keywords: Mechanotransduction, Substrate Stiffness, 3D Culture, Tissue Engineering, Stem Cell Differentiation 


\begin{abstract}
The role of substrate mechanics in guiding mesenchymal stem cell (MSC) fate has been the focus of much research over the last decade. More recently, the complex interplay between substrate mechanics and other material properties such as ligand chemistry and substrate degradability to regulate MSC differentiation has begun to be elucidated. Additionally, there are several changes in the presentation of these material properties as the dimensionality is altered from two- to three-dimensional substrates, which may fundamentally alter our understanding of substrate-induced mechanotransduction processes. In this review, an overview of recent findings that highlight the material properties that are important in guiding MSC fate decisions is presented, with a focus on underlining gaps in our existing knowledge and proposing potential directions for future research.
\end{abstract}




\section{Introduction}

Mesenchymal stem cells (MSCs) isolated from bone marrow have great potential as a cell source for regenerative medicine due to both their relative ease of isolation and their ability to undergo differentiation towards multiple lineages (1-3). Initially, the use of biochemical factors to induce controlled differentiation was seen as a key aspect of their effective clinical translation (4). However, over the last decade there has been much research carried out on the role of the material properties of the substrates that MSCs are seeded onto, or embedded within, in guiding differentiation. While substrate mechanics has long been known to have an impact on cellular activity (5), a seminal manuscript by Engler et al. first provided evidence that MSC differentiation could be directed by substrate mechanics (6). This discovery brought about renewed interest in the field of cellular mechanotransduction, with much of the research focused on characterizing the cellular signaling mechanisms involved in sensing and responding to two-dimensional substrate stiffness. In this review, we do not cover the various different cellular processes thought to be involved in mechanotransduction, but point the interested reader to several excellent reviews on this topic $(5,7-9)$. Instead, here we focus on the material parameters that are known to impact cellular mechanotransduction and present these as design choices that must be carefully considered when developing a biomaterials-based study. To illustrate the importance of these material parameters, in Section 2 we introduce the molecular clutch hypothesis, as it provides an excellent framework within which to explore the role of biomaterials design choices. 
The majority of existing research has focused on the role of substrate stiffness in guiding cell fate. Conversely, the relative importance of other material properties and how they may interact with stiffness cues has seen less focus, leaving some gaps in our knowledge and presenting opportunities for further discoveries. It has become increasingly apparent that elastic modulus alone is not the sole material property governing the mechanotransduction response of MSCs and that there is significant interplay between mechanics and properties such as ligand chemistry and substrate degradation (10-14). Additionally, the majority of existing research has been carried out using two dimensional (2D) substrates. Mechanotransduction is likely to be inherently different in the three dimensional (3D) environments employed in most therapeutic strategies using MSCs (15). In the few studies that have investigated MSC response to 3D materials, there have been notable differences in comparison to behavior on $2 \mathrm{D}$ surfaces $(11,12,16)$. As a result, there is a need for investigation of MSC response to material properties in 3D substrates, particularly in macro-porous environments, which are considerably more complex from a topographical and mechanical viewpoint (17). Furthermore, due to the complexity of separating material variables in such experiments, novel materials science approaches are required to enable single variable studies to reveal their relative importance and potential non-additive outcomes.

In this review, we summarize recent findings that highlight the importance of materials in guiding MSC fate decisions, underline gaps in our existing knowledge, and propose potential directions for future research. First, we describe the development of myosinmediated traction, which is the basic mechanism underlying cellular mechanosensation of substrate mechanics. Following this, we highlight several specific material properties 
that recent studies have revealed to be important in directing MSC differentiation. To conclude the review, we discuss new materials strategies and experimental techniques that have the potential to lead to an increased understanding of these phenomena towards the ultimate goal of engineering effective regenerative medicine therapies.

\section{Cellular Mechanotransduction of Material Stiffness}

In order to appreciate the importance of material properties in directing MSC fate, it is first necessary to understand the basic principals by which cells sense and respond to their local mechanical environment, a process which is termed mechanotransduction (Fig. 1). In this context, the dominant mechanism proposed in the field is that cells sense the stiffness or rigidity of the surrounding substrate through integrin-ligand attachments (8). At the interface between cells and the substrate, ligands presented on the surface of the substrate are recognized and engaged by integrin receptors located at the cell membrane, which in turn enables the binding of these integrins to the actin cytoskeleton within the cell. This results in a tensile force at the cell-substrate interface, as the actin that forms the cytoskeleton is constantly flowing towards the center of the cell due to the action of myosin motors in a process termed retrograde flow $(18,19)$. The tension developed at this interface is proportional to the resistance provided by the substrate, and if the tension generated is high enough, the adhesion can mature into what is known as a focal adhesion complex. Both the development of tension and the maturation of focal adhesion complexes are believed to trigger the signaling processes that alter cellular activity including spreading, migration and differentiation $(8,18)$. 
Importantly, several models have been developed to capture the complexity of the interactions at this interface and to provide a better understanding of the material properties that are key to defining the tensile forces developed by cells (20). The most prominent of these models is based on the hypothesis that the integrin-ligand interface acts as a molecular 'clutch' (21-23). In this model, myosin motors pull an actin filament rearward towards the 'center' of a cell with a force $\mathrm{F}_{\text {Myosin }}$ and at a velocity Vilament (Fig. 1). Integrin-ligand clutches reversibly and stochastically engage and disengage at rates $\mathrm{k}_{\mathrm{on}}$ and $\mathrm{k}_{\mathrm{off}}$, respectively, and generate resistance to the rearward flow of actin. This causes stretching of the integrin-ligand clutches and their eventual failure at a force dependent rate $\mathrm{k}_{\mathrm{off}}$ * The forces developed by this process are balanced by deformation of the substrate, resulting in a substrate strain $\mathrm{X}_{\text {Sub. Therefore, resistance to }}$ loading and resulting tension developed at the adhesion is defined by both the stiffness of the bound clutches $\mathrm{K}_{\text {Clutch }}$ and the substrate stiffness $\mathrm{K}_{\text {Sub }}(22)$. In this framework, the stiffness of the clutches is defined by the number, stiffness and binding rates of integrin-ligand bonds, which in turn is determined by the density and identity of the ligands present on the material surface and the cell type. This results in a complex relationship between cellular tension and substrate stiffness, with several different modes of behavior $(22,24)$. However, from a materials perspective, the important variables defining cellular mechanotransduction can be identified as material stiffness, ligand density and ligand identity. It is also worth noting that additional factors such as dimensionality can have profound effects on the presentation of these properties to the cells, as will be further discussed in section 3.4.

\section{Material Properties and MSC Differentiation}


In this section, we highlight recent studies that have advanced our understanding of the relationship between material properties, cellular mechanotransduction and MSC differentiation. Initially, we discuss intrinsic material properties, before moving on to discussing the changes brought about by transitioning dimensionality from 2D to 3D.

\subsection{Substrate Stiffness}

Engler and co-authors were the first to demonstrate that MSC fate could be guided by substrate mechanics (6). They seeded MSCs onto collagen-coated polyacrylamide substrates with different levels of stiffness and assayed for markers of differentiation (morphology, gene transcription and protein expression). The results revealed that MSCs show markers for neurogenic lineages on low stiffness substrates (0.1-1 kPa), myogenic lineages at intermediate stiffness $(8-17 \mathrm{kPa})$ and osteogenic lineages at the highest stiffness $(25-40 \mathrm{kPa})$. Interestingly, after three weeks of stiffness-directed differentiation, the lineage commitment of MSCs was found to be irreversible even upon the addition of soluble factors known to initiate an alternative differentiation pathway. Several studies replicating the basic premise of this manuscript have confirmed the role of substrate stiffness in guiding the differentiation of MSCs (25-29). These later experiments have typically focused on differentiation towards adipogenic and/or osteogenic lineages, as the ability of MSCs to fully commit to other differentiated cell types continues to be somewhat contentious (1).

Yang et al. made a notable contribution to this area of research when they pre-cultured cells on photodegradable hydrogels and tissue-culture plastic (TCP) to demonstrate that the substrate mechanics of an initial substrate could bias MSC fate decisions even after 
transition to a new substrate (26). Pre-culture on TCP (3 GPa) for periods longer than 1 day resulted in a bias in MSC differentiation towards osteogenic over adipogenic lineages after a transition to a soft $(2 \mathrm{kPa})$ polyethylene glycol (PEG) hydrogel. A variant of this experiment was also carried out using stiff (10 kPa) hydrogels that could be weakened to $2 \mathrm{kPa}$ by UV degradation of hydrogel crosslinks. Both 1 and 7 days of culture on $10 \mathrm{kPa}$ substrates resulted in an up-regulation of RUNX2 expression (a marker of osteogenic differentiation) that was reversed 5 days post softening of the hydrogels to $2 \mathrm{kPa}$. However, softening the hydrogels after the MSCs were cultured in stiff conditions for 10 days could no longer reverse the up-regulation of RUNX2 expression. This work has important implications for maintaining the differentiation potential of MSCs during isolation, expansion and eventual therapeutic delivery.

In the studies described above, MSCs are induced to adhere to material substrates either through coating of the polymeric substrate with an extracellular matrix protein such as collagen or through the tethering of integrin-adhesive ligands. It has been suggested that the methods used to modify the stiffness of polyacrylamide substrates for MSC differentiation studies may also alter the substrate mesh size, causing a reduction in the average distance between the collagen tethering points to the underlying substrate (30). Because cells adhere directly to the collagen and not the underlying substrate, Trappmann et al. proposed that this reduction in tethering distance may result in an increase in collagen fiber stiffness to which MSCs could respond independent from the underlying substrate properties. This hypothesis was based on the observation that substrate stiffness did not influence MSC differentiation upon polydimethylsiloxane (PDMS) substrates. However, a subsequent study by Wen et al. noted that the 
mechanical characterization of the PDMS carried out previously did not fully account for the strain rate dependence of the modulus (28). At rates relevant to cell-substrate interactions, all of the PDMS substrates used by Trappmann et al. had stiffness values that would be considered stiff $(>30 \mathrm{kPa})$. Nevertheless, in cases where the tethered adhesive ligand or protein is more compliant than the substrate to which it is anchored, it is possible that the tethering itself is an important factor in mediating MSC differentiation (31). Such cases exist in orthopaedic and dental biomaterials applications where protein coatings are frequently tethered to metallic substrates (32, 33). Further research on this aspect of cellular mechanotransduction is required to complete our understanding of cellular response to substrate mechanics (31). This will be discussed in more detail in Section 3.3 below.

\subsection{Viscoelasticity}

In the previous section, the majority of the work discussed had assumed that the substrates presented to MSCs were purely elastic and strain rate independent. However, many of the natural and synthetic polymers used as substrates for cell culture are viscoelastic materials, meaning they exhibit both elastic and viscous characteristics when loaded $(23,28,34)$. Notably, native biological materials that comprise mammalian tissue are also viscoelastic $(35,36)$. Viscoelastic substrates are commonly characterized using rheometry with the shear modulus split into elastic (storage modulus, G') and viscous (loss modulus, G") components. An important aspect of viscoelasticity is creep, a time-dependent reduction in stiffness when exposed to a constant applied force. In the context of mechanotransduction, substrate creep would be expected to result in decreasing substrate stiffness over time. Despite the potential 
impact of viscoelasticity on MSC behavior, few studies have focused on this aspect of substrate mechanics $(13,14)$.

Cameron et al. seeded MSCs on collagen-coated polyacrylamide substrates with a constant storage modulus of $4.7 \mathrm{kPa}$ and varying loss moduli over a range of 1-130 $\mathrm{Pa}$ (13). A significant increase in cell-spread area and proliferation was observed upon the substrates with the largest loss modulus. Interestingly, increased cell spreading was not associated with an increased number of mature focal adhesions. Furthermore, when cultured with the corresponding differentiation induction media, MSCs showed increased differentiation towards adipogenic, myogenic and osteogenic lineages upon the increased loss modulus substrates. A follow-up study linked increased loss modulus with an increase in cellular protrusions and Rac1 activation, potentially explaining the increased cell spreading (14). Rac1 is a small signaling protein that upon activation leads to an increase in actin polymerization, thus resulting in protrusions at the leading edge of cells (37). The authors propose that this behavior can be explained by the cellular tensegrity model (20), stating that viscoelastic flow results in a loss of tension in the actin cytoskeleton that is balanced by an increase in the active tension of the cell through spreading. However, these results are also consistent with those of Chaudhuri et al. who observed increased osteosarcoma cell spreading on viscoelastic alginate hydrogels (23). These authors used computational models based on molecular clutches to propose the hypothesis that increased spreading was due to the clustering of ligands through viscoelastic flow of the substrate. Thus, it is clear that viscoelasticity plays a significant role in MSC response to substrate mechanics and that further work is needed to determine the specific mechanisms. Indeed, as discussed in the previous section, a 
failure to account for viscoelasticity may explain some discrepancies in the substrateinduced mechanotransduction literature $(28,30)$.

\subsection{Ligand Identity and Density}

Alongside research demonstrating the importance of substrate mechanics there also have been several recent studies indicating a role for ligand identity and density in directing MSC differentiation (10, 29, 38-40). Ligands are short amino acid sequences that are present in extracellular matrix (ECM) proteins. These sequences are recognized and form attachments with specific integrins depending on their amino acid composition (41). Biological substrates such as collagen, laminin and fibronectin intrinsically contain adhesive ligands, whereas synthetic polymers are typically functionalized by chemically linking small peptides that contain the adhesive ligand sequence to the polymer backbone. An advantage of synthetic systems is that the ligand identity and density can be tightly controlled, whereas the properties of native ECM proteins are less well defined. In the context of the mechanotransduction response of cells to a substrate, ligand density and identity will determine the number, stiffness and binding rates of integrin-ligand bonds $(22,42)$.

With this in mind, several studies have investigated the effect of ligand identity on MSC differentiation. Rowlands et al. used polyacrylamide substrates that were coated with different ECM proteins (collagen I, collagen IV, laminin and fibronectin) in order to determine the interplay between stiffness $(0.7-80 \mathrm{kPa})$ and ligand presentation on human MSC differentiation towards myogenic or osteogenic lineages (29). Significant expression of osteogenic differentiation markers was only observed on the stiffest 
substrates when coated with collagen I. In contrast, up-regulation of myogenic differentiation markers was observed on fibronectin-coated substrates at $25 \mathrm{kPa}$ and on collagen I-coated substrates at $80 \mathrm{kPa}$. The use of different ECM proteins in this study emulates an important aspect of the native environment of cells. However, while the authors confirmed that the quantities of tethered protein were consistent across the different ECM proteins used, the ligand densities are not likely to be consistent between the different ECM coatings. This is an inherent feature of comparisons between ECM proteins due to differences in protein molecular weight and number of ligands per protein molecule. Nonetheless, the fact that osteogenic differentiation was only observed on collagen I-coated hydrogels suggests that in some cases specific ligands are required to activate particular differentiation pathways.

Frith et al. used a more controlled experimental approach by functionalizing a polystyrene-block-poly(ethylene oxide)-copolymer substrate with short ligand peptides: RGD, IKVAV, YIGSR and RETTAWA (10). The stiffness of the substrates was not altered but the laminin-derived IKVAV peptide was observed to support increased osteogenesis, whereas both IKVAV and RETTAWA supported adipogenesis. Additionally, it was noted that a minimum level of RGD was required for the viability of MSCs beyond initial attachment. Furthermore, the expression of specific integrins on the cell membrane was found to transiently increase as cells differentiated towards different lineages, with integrin subunit $\alpha 5$ increasing during osteogenesis and integrin $\alpha 6$ increasing during adipogenesis. The absence of any collagen-mimetic ligand sequences was a limitation of the study, as Mehta et al. observed increased levels of MSC differentiation towards an osteogenic lineage when the cells were seeded onto 
alginate hydrogels functionalized with the collagen-mimetic ligand DGEA compared to hydrogels functionalized with RGD (40). Interestingly, this effect was only observed when the cells were encapsulated within a 3D hydrogel and not when they were seeded onto $2 \mathrm{D}$ surfaces.

Comparatively, there are few studies on the effect of ligand density on MSC differentiation. However, Luo et al. have demonstrated that adipogenic differentiation is dependent on the both the density and identity of ligands using novel alkanethiolate substrates in a microarray culture system (38). Huebsch et al. also observed an effect of ligand density on the strain range that induces MSCs to commit to osteogenic lineages, with a decrease in ligand density resulting in reduced osteogenic differentiation at intermediate stiffness values (11). Additionally, the lateral spacing of RGD has also been demonstrated to effect the differentiation of MSCs; osteogenesis was increased at lower ligand spacing, whereas adipogenesis was increased as the spacing between RGD ligands increased (39). Finally, the spreading of osteosarcoma cells in response to substrate stiffness also has been demonstrated to be dependent on ligand density, with an increase in density producing a similar response to increasing substrate stiffness (23). Computational models of cell-substrate interactions predict these observed effects of ligand density on cellular behavior $(22,24)$. In essence, an increase in ligand density results in a greater number of bonds between the substrate and the cell, thereby increasing the resistance to actin retrograde flow and thus increasing the substrate rigidity that is sensed by the cell. Moreover, a parameter sensitivity analysis of the clutch model reveals that cellular tension is potentially more sensitive to ligand density 
than substrate stiffness (24), suggesting that this key variable could be further exploited in engineered substrates for MSC regenerative therapies.

\subsection{Dimensionality and Degradation}

As 3D culture is an essential aspect of many approaches in regenerative medicine, an understanding of the differences that occur as material dimensionality shifts from $2 \mathrm{D}$ surfaces to 3D substrates is highly important (15). Broadly speaking, 3D culture systems can be split into two distinct groups; macro-porous and non-macro-porous substrates, where a "macro" sized pore is on the length scale of a single cell or greater ( 10 microns). There are important differences between these types of substrates that alter the relationship between material properties and MSC differentiation. In a macroporous substrate, the pore geometry and architecture can have a significant effect on cellular behavior (17). In contrast, in non-porous substrates, the relationship between stiffness and the ability of cells to degrade the hydrogel becomes significant $(12,43)$. In this section we discuss the differences between the two types of 3D culture systems and their effects on the role of material properties in guiding MSC fate decisions.

In the case of macro-porous substrates, commonly the cellular mechanical environment is characterized by reporting either the modulus of the bulk porous structure or the modulus of the constituent material without pores $(16,44,45)$. However, Harley et al. noted that in macro-porous 3D scaffolds, the rigidity of the pore struts on which the cells are grown may be a more relevant parameter for impacting cellular behavior (17). Indeed, in macro-porous scaffolds with pore sizes on the order of $100 \mu \mathrm{m}$, cells have been observed to bridge pores and generate tension perpendicular to the strut geometry 
causing buckling (46-48). Furthermore, what perhaps has not been fully appreciated is that the macro-porous nature of these scaffolds results in the exposure of cells to a heterogeneous mechanical environment. This is a result of the ability of cells to assume a multitude of different orientations within a porous structure. The local stiffness presented to the cell is then a function both of the exact adhesion location and of the orientation of tensile force with respect to the pore surface. For example, a cell pulling perpendicular to the material surface at the mid-span of a strut will sense less resistance than a cell pulling closer to a strut base (Fig. 2). As a result of this structural complexity, computational models are required to fully characterize the range of substrate mechanical properties to which cells are exposed within porous materials.

Marklein et al. have performed one of the most extensive studies of the effect of porous substrate stiffness on MSC fate (16). Porous, methacrylated hyaluronic acid hydrogels (MeHA) were fabricated using a sacrificial poly(methyl methacrylate) (PMMA) microsphere template. The Young's modulus of the porous substrates (1.5-7.4 $\mathrm{kPa})$ was calculated using the modulus of non-porous MeHA hydrogels and the Gibson-Ashby model for open cell foams (49). Analysis of gene expression after 14 days of culture in growth media revealed that some markers of adipogenic and osteogenic differentiation were up-regulated on the weakest substrates, possibly due to increased cell-cell contact caused by cell-mediated contraction of the porous structure. Additionally, myogenic differentiation was significantly down-regulated upon the weakest substrates. Murphy et al. investigated rat MSC differentiation towards either chondrogenic or osteogenic lineages within highly porous, collagen-glycosaminoglycan scaffolds, with the Young's modulus of the porous substrates ranging from $0.5-1.5 \mathrm{kPa}(44)$. Analysis of MSC gene 
expression revealed significant increases in RUNX2, an early stage marker of osteogenic differentiation, within the stiffest substrates after 7 days in growth media. Additionally, a marker of chondrogenic differentiation, Sox9, was significantly greater within the weakest substrates by day 3 . Her et al. investigated the differentiation of MSCs towards neural lineages using similar collagen-based substrates (50). In supplemented neurobasal medium, MSCs were found to differentiate towards glial lineages on the stiffest substrates $(10 \mathrm{kPa})$ and neuronal lineages on the least stiff substrates $(1 \mathrm{kPa})$. Wingate et al. investigated MSC differentiation towards endothelial or smooth muscle-like lineages upon electrospun, polyethylene glycol dimethacrylate substrates with Young's moduli ranging from 2-15 kPa (51). Significant levels of endothelial differentiation were observed upon the weakest substrates within 24 hours in growth media, whereas markers of smooth muscle-like differentiation were observed in $80 \%$ of cells seeded upon substrates with moduli greater than $8 \mathrm{kPa}$.

Aside from differences in culture conditions, pore structure and material composition, there are additional factors that make comparisons between these studies difficult. It is possible, for example, that the inherent heterogeneity of the macro-porous mechanical stimulus obscures any clear trends in MSC differentiation when observed from pooled gene expression data alone. An additional complication of quantifying differentiation through analysis of gene expression is the risk of missing transient up- or downregulation by not selecting appropriate time points. These challenges in assessing stiffness-directed differentiation in 3D macro-porous substrates may be reduced through the use of complementary histological assays. However, while direct comparisons between studies are difficult, taken together the data clearly demonstrate 
that the important role of substrate mechanics in guiding stem cell fate is maintained in transitioning from 2D to 3D macro-porous substrates.

Perhaps the greatest changes in moving from 2D to 3D systems are observed within non-macro-porous substrates. 2D culture induces cell polarity, which is typically retained in 3D macro-porous substrates where the pores are sufficiently large to represent a curved 2D surface on the scale of a cell. However, when cells are fully encapsulated within non-macro-porous 3D substrates, they are exposed to conformal cell-substrate contact. This can induce the cells to assume a stellate, isotropic morphology and often cell polarization is only realized upon initiation of migration (15). Additionally, cells must first degrade the surrounding substrate in order to migrate or probe their surroundings (43). Furthermore, the nature of non-macro-porous substrates can result in considerable gradients of soluble factors throughout the substrate, which are dependent on the substrate mesh size. Finally, a further complication in determining the role of these variables on cellular activity is that the methods used to alter mechanics, such as covalent crosslinking, may also change the degradability and mesh size of the substrate, making it challenging to carry out single variable studies $(52,53)$.

Huebsch et al. encapsulated MSCs within non-macro-porous, alginate hydrogels functionalized with RGD ligand motifs and assessed the effect of stiffness on both osteogenic and adipogenic differentiation (11). Interestingly, osteogenic differentiation was found to occur predominantly within substrates of intermediate stiffness (11-30 $\mathrm{kPa}$ ), whereas adipogenic differentiation was observed within the weakest substrates 
(2.5-5 kPa, Fig. 3A). Contrary to $2 \mathrm{D}$ studies, differences in differentiation and substrate mechanics were not correlated with changes in cellular morphology (Fig. 3B). The authors demonstrated that integrin engagement with the RGD ligands was altered in $3 \mathrm{D}$, revealing that integrin $\alpha \mathrm{v}$ was responsible for $2 \mathrm{D}$ substrate adhesion, whereas both integrin $\alpha_{V}$ and $\alpha_{5}$ were engaged in 3D substrate adhesion. Similarly, changes in integrin engagement with DGEA ligands have been observed as a result of transitioning to $3 \mathrm{D}$ culture (40). Furthermore, the maximum level of integrin binding and clustering correlated with the maximum commitment to osteogenic differentiation at intermediate substrate stiffness $(22 \mathrm{kPa})$. These differences in integrin-ligand attachments in 2D compared to 3D demonstrate the importance of further 3D substrate studies to promote the development of MSC-based regenerative medicine therapies.

Interestingly, the stiffness of the alginate hydrogels used by Huebsch et al. was controlled through a physical crosslinking process using interactions between alginate and calcium ions. Physical crosslinks are bonds formed though non-covalent mechanisms such as ionic and hydrophobic interactions, which are often weak enough to be broken by the forces generated by cells and can reform or repair after breaking. It has been postulated that the reversible nature of these crosslinks allows the MSCs to remodel the surrounding substrate and that MSC behavior within these hydrogels would likely be very different in comparison to covalently-crosslinked hydrogels (12). In order to further address these questions, Khetan et al. investigated the role of degradation-mediated traction in directing MSC fate within covalently-crosslinked MeHA hydrogels (12). They found that within non-macro-porous MeHA hydrogels, MSCs predominantly underwent adipogenesis independent of the value of substrate 
stiffness (4-91 kPa). However, when cells were seeded on top of the substrates (i.e., 2D) rather than encapsulated within (i.e., 3D), osteogenesis was observed at similar stiffness values to those of Huebsch and coauthors (25 kPa). MSCs were then placed in modified MeHA hydrogels that were matrix metalloproteinase (MMP) degradable, with UV-triggered shielding of the MMP degradable sequences (Fig. 3C-G). In 3D cultures, significant levels of osteogenic differentiation and corresponding changes in cellular morphology were observed within the degradable hydrogels in comparison to non-

degradable hydrogels. This work demonstrates that matrix degradation is necessary for cellular mechanotransduction-driven fate decisions when MSCs are encapsulated within covalently-crosslinked, non-macro-porous hydrogels.

\section{Future Opportunities and Conclusions}

Despite the growing body of data demonstrating that material properties have a strong role in guiding MSC fate, there are still no guidelines for the design of therapeutically relevant, 3D materials to direct differentiation towards specific lineages. This is partly explained by the complex interaction between variables such as ligand chemistry, substrate mechanics and dimensionality in defining the mechanical environment presented to MSCs and the difficulty in separating these variables. In order to overcome this limitation, new material platforms that enable independent control of substrate properties are needed. Simultaneously, there is also a need for novel experimental techniques that allow nondestructive characterization of material properties and cellular behavior within 3D systems. 
Natural, protein-based materials are not well suited for detailed mechanistic studies of MSC fate, as the ligand identity and density cannot be varied in a controlled manner. Additionally, the chemistry used to crosslink such hydrogels can often obscure cellbinding ligands $(53,54)$. An alternative approach may be recombinant synthesis of engineered proteins, which can be used to produce protein-based substrates with independent control of stiffness and ligand chemistry $(52,55)$. Furthermore, these engineered proteins can be modified to include proteolytic target sites that enable control of degradation (56). Non-protein-based hydrogels, including alginate, hyaluronic acid and PEG are also candidate materials for future MSC fate studies (16, 23, 26). However, when modifying these materials to include cell-adhesive ligands, care must be taken to ensure potential effects on mechanics are taken into account (31, $57)$.

Regardless of the choice of polymer backbone, novel crosslinking and conjugation chemistries that are well controlled and cell-compatible will greatly enhance the study of substrate-induced MSC differentiation. For example, orthogonal crosslinking techniques such as the use of click chemistry present an opportunity to alter the mechanics of hydrogels in a cytocompatible manner without the confounding issue of cross-reactions with cell-binding ligands (54, 58-60). Additionally, Kakinoki et al. have recently studied endothelial cell mechanotransduction using substrates consisting of RDG ligands tethered to ring shaped $\alpha$-cyclodextrins ( $\alpha$-CDs) which were threaded by a PEG chain (61). As the $\alpha$-CDs are able to slide and rotate on the PEG chain, this allowed the ligands mobility independent of substrate stiffness. The combination of this material with immobile ligands may allow the biochemical and mechanical effects of 
ligand density/clustering to be separated. We previously highlighted the important insights obtained through the use of materials with degradation rates and mechanics that could be directly modified at specific time points $(12,26,62)$. Recently, methods have been developed that allow ligand density to be controlled in a similar fashion by protecting them with caging groups that are released upon exposure to ultraviolet light (63). Similarly, Wylie et al. have fabricated 3D agarose hydrogels with coumarin-caged thiol groups (64). Subsequent uncaging of the thiol groups using two-photon irradiation and reaction with binding peptides allowed the spatial control of peptide conjugation in distinct volumes and gradients. This technique has the potential to allow the fabrication of 3D hydrogels with spatially controlled ligand distribution and thus investigation of the effect of ligand patterning induced cell polarization on differentiation. The application of these materials to studies of substrate-guided MSC differentiation has exciting potential.

In addition to advances in novel biomaterials design, advances in biomaterials characterization are also required to propel the field forward. There are several new experimental techniques that have exciting potential for researchers interested in studying cellular activity and cell-substrate interactions (65). In particular, techniques that allow non-destructive monitoring within 3D environments are required to facilitate experiments using a wide range of material permutations to be carried out with a reasonable sample size. For example, coherent anti-Stokes Raman scattering (CARS) microscopy allows 3D live imaging of lipid-rich structures within cells (66). In addition, combination of CARS with second harmonic generation (SHG) or spectral ultrasound imaging (SUSI) may be used to assess markers of differentiation within 3D 
substrates in a non-destructive manner, as CARS can identify the build up of lipids associated with adipogenesis and SHG or SUSI can be used to quantify collagen deposition and mineralization associated with osteogenic differentiation (67-69). To assess the mechanical environment, 3D traction force microscopy (TFM) allows quantification of the tensile forces that cells apply to the surrounding substrate. This technique has been used to provide a direct measure of the tension developed by cells in response to the degradability of their local environment (12). Similarly, microrheology allows characterization of the local mechanical properties around individual cells encapsulated within 3D substrates and can be used to quantify cell-mediated degradation of the surrounding material $(43,70)$. Finally, the combination of advances in microscopy and materials science with high-throughput array-based techniques provides the capability to allow researchers to systematically gather data from a wide range of substrate permutations, potentially leading to a comprehensive understanding of substrate-MSC interactions $(71,72)$.

In this review, we have discussed substrate-driven MSC differentiation and it's implications in the design of materials for use in regenerative medicine. Despite the advances in our understanding made by existing research in this area, it's currently unclear how much or if substrate properties matter when cells and materials are placed in vivo. This topic has only recently begun to be explored and is likely to become an emerging new research direction for the field (73). Consequently, future research focused on expanding our knowledge of substrate-driven differentiation must also be coupled with experiments focused on potential translation of the findings to new therapeutic strategies. 


\section{Acknowledgements}

The authors would like to thank Dr. Ted Vaughan and Dr. Laoise McNamara (NUI

Galway, Ireland) for insightful discussion. The authors acknowledge funding provided

by NIH R21 EB018407-01, NIH U19 AI116484-01, NIH R21 EB020235-01, NSF

DMR 1508006, California Institute for Regenerative Medicine RT3-07948, and Stanford University SICB-112878, Bio-X IIP-7-75 (S.C.H.), and an Irish Research Council ELEVATE fellowship ELEVATEPD/2013/30 (M.H.).

\section{References:}

1. Uccelli A, Moretta L, Pistoia V. Mesenchymal stem cells in health and disease. Nat Rev Immunol. 2008; 8(9):726-36.

2. Caplan AI. Mesenchymal stem cells. J Orthop Res. 1991; 9(5):641-50.

3. Pittenger MF, Mackay AM, Beck SC, Jaiswal RK, Douglas R, Mosca JD, et al. Multilineage potential of adult human mesenchymal stem cells. Science. 1999; 284(5411):143-7.

4. Murphy WL, McDevitt TC, Engler AJ. Materials as stem cell regulators. Nat Mater. 2014; 13(6):547-57.

5. Iskratsch T, Wolfenson H, Sheetz MP. Appreciating force and shape-the rise of mechanotransduction in cell biology. Nat Rev Mol Cell Biol. 2014; 15(12):825-33.

6. $\quad$ Engler AJ, Sen S, Sweeney HL, Discher DE. Matrix elasticity directs stem cell lineage specification. Cell. 2006; 126(4):677-89.

7. Parsons JT, Horwitz AR, Schwartz MA. Cell adhesion: integrating cytoskeletal dynamics and cellular tension. Nat Rev Mol Cell Biol. 2010; 11(9):633-43.

8. Puklin-Faucher E, Sheetz MP. The mechanical integrin cycle. J Cell Sci. 2009; 122(Pt 2):179-86.

9. Fedorchak GR, Kaminski A, Lammerding J. Cellular mechanosensing: getting to the nucleus of it all. Prog Biophys Mol Biol. 2014; 115(2-3):76-92.

10. Frith JE, Mills RJ, Hudson JE, Cooper-White JJ. Tailored integrin-extracellular matrix interactions to direct human mesenchymal stem cell differentiation. Stem Cells Dev. 2012; 21(13):2442-56.

11. Huebsch N, Arany PR, Mao AS, Shvartsman D, Ali OA, Bencherif SA, et al. Harnessing traction-mediated manipulation of the cell/matrix interface to control stemcell fate. Nat Mater. 2010; 9(6):518-26.

12. Khetan S, Guvendiren M, Legant WR, Cohen DM, Chen CS, Burdick JA. Degradation-mediated cellular traction directs stem cell fate in covalently crosslinked three-dimensional hydrogels. Nat Mater. 2013; 12(5):458-65. 
13. Cameron AR, Frith JE, Cooper-White JJ. The influence of substrate creep on mesenchymal stem cell behaviour and phenotype. Biomaterials. 2011; 32(26):5979-93. 14. Cameron AR, Frith JE, Gomez GA, Yap AS, Cooper-White JJ. The effect of time-dependent deformation of viscoelastic hydrogels on myogenic induction and Rac1 activity in mesenchymal stem cells. Biomaterials. 2014; 35(6):1857-68.

15. Baker BM, Chen CS. Deconstructing the third dimension: how 3D culture microenvironments alter cellular cues. J Cell Sci. 2012; 125(Pt 13):3015-24.

16. Marklein RA, Soranno DE, Burdick JA. Magnitude and presentation of mechanical signals influence adult stem cell behavior in 3-dimensional macroporous hydrogels. Soft matter. 2012; 8(31):8113-20.

17. Harley BAC, Kim H-D, Zaman MH, Yannas IV, Lauffenburger DA, Gibson LJ. Microarchitecture of Three-Dimensional Scaffolds Influences Cell Migration Behavior via Junction Interactions. Biophys J. 2008; 95(8):4013-24.

18. Case LB, Waterman CM. Integration of actin dynamics and cell adhesion by a three-dimensional, mechanosensitive molecular clutch. Nat Cell Biol. 2015; 17(8):95563.

19. Moore SW, Roca-Cusachs P, Sheetz MP. Stretchy proteins on stretchy substrates: the important elements of integrin-mediated rigidity sensing. Dev Cell. 2010; 19(2):194-206.

20. Ingber DE. Tensegrity I. Cell structure and hierarchical systems biology. J Cell Sci. 2003; 116(Pt 7):1157-73.

21. Mitchison T, Kirschner M. Cytoskeletal dynamics and nerve growth. Neuron. 1988; 1(9):761-72.

22. Chan CE, Odde DJ. Traction dynamics of filopodia on compliant substrates. Science. 2008; 322(5908):1687-91.

23. Chaudhuri O, Gu L, Darnell M, Klumpers D, Bencherif SA, Weaver JC, et al. Substrate stress relaxation regulates cell spreading. Nat Commun. 2015; 6:6364.

24. Bangasser BL, Rosenfeld SS, Odde DJ. Determinants of maximal force transmission in a motor-clutch model of cell traction in a compliant microenvironment. Biophys J. 2013; 105(3):581-92.

25. Fu J, Wang YK, Yang MT, Desai RA, Yu X, Liu Z, et al. Mechanical regulation of cell function with geometrically modulated elastomeric substrates. Nat Methods. 2010; 7(9):733-6.

26. Yang C, Tibbitt MW, Basta L, Anseth KS. Mechanical memory and dosing influence stem cell fate. Nat Mater. 2014; 13(6):645-52.

27. Dupont S, Morsut L, Aragona M, Enzo E, Giulitti S, Cordenonsi M, et al. Role of YAP/TAZ in mechanotransduction. Nature. 2011; 474(7350):179-83.

28. Wen JH, Vincent LG, Fuhrmann A, Choi YS, Hribar KC, Taylor-Weiner H, et al. Interplay of matrix stiffness and protein tethering in stem cell differentiation. Nat Mater. 2014; 13(10):979-87.

29. Rowlands AS, George PA, Cooper-White JJ. Directing osteogenic and myogenic differentiation of MSCs: interplay of stiffness and adhesive ligand presentation. Am J Physiol Cell Physiol. 2008; 295(4):C1037-44.

30. Trappmann B, Gautrot JE, Connelly JT, Strange DG, Li Y, Oyen ML, et al. Extracellular-matrix tethering regulates stem-cell fate. Nat Mater. 2012; 11(7):642-9.

31. Chaudhuri O, Mooney DJ. Stem-cell differentiation: Anchoring cell-fate cues. Nat Mater. 2012; 11(7):568-9. 
32. Raphel J, Karlsson J, Galli S, Wennerberg A, Lindsay C, Haugh MG, et al. Engineered protein coatings to improve the osseointegration of dental and orthopaedic implants. Biomaterials. 2016; 83:269-82.

33. Bronk JK, Russell BH, Rivera JJ, Pasqualini R, Arap W, Hook M, et al. A multifunctional streptococcal collagen-mimetic protein coating prevents bacterial adhesion and promotes osteoid formation on titanium. Acta Biomater. 2014; 10(7):3354-62.

34. Murrell M, Kamm R, Matsudaira P. Substrate viscosity enhances correlation in epithelial sheet movement. Biophys J. 2011; 101(2):297-306.

35. Ahearne M, Yang Y, El Haj AJ, Then KY, Liu KK. Characterizing the viscoelastic properties of thin hydrogel-based constructs for tissue engineering applications. J R Soc Interface. 2005; 2(5):455-63.

36. Krishnan L, Weiss JA, Wessman MD, Hoying JB. Design and application of a test system for viscoelastic characterization of collagen gels. Tissue Eng. 2004; 10(12):241-52.

37. Burridge K, Wennerberg K. Rho and Rac take center stage. Cell. 2004; 116(2):167-79.

38. Luo W, Chan EW, Yousaf MN. Tailored electroactive and quantitative ligand density microarrays applied to stem cell differentiation. J Am Chem Soc. 2010; 132(8):2614-21.

39. Frith JE, Mills RJ, Cooper-White JJ. Lateral spacing of adhesion peptides influences human mesenchymal stem cell behaviour. J Cell Sci. 2012; 125(Pt 2):31727.

40. Mehta M, Madl CM, Lee S, Duda GN, Mooney DJ. The collagen I mimetic peptide DGEA enhances an osteogenic phenotype in mesenchymal stem cells when presented from cell-encapsulating hydrogels. J Biomed Mater Res A. 2015:n/a-n/a. 41. Humphries JD, Byron A, Humphries MJ. Integrin ligands at a glance. J Cell Sci. 2006; 119(Pt 19):3901-3.

42. Elosegui-Artola A, Bazellieres E, Allen MD, Andreu I, Oria R, Sunyer R, et al. Rigidity sensing and adaptation through regulation of integrin types. Nat Mater. 2014; 13(6):631-7.

43. Schultz KM, Kyburz KA, Anseth KS. Measuring dynamic cell-material interactions and remodeling during 3D human mesenchymal stem cell migration in hydrogels. Proc Natl Acad Sci U S A. 2015; 112(29):E3757-64.

44. Murphy CM, Matsiko A, Haugh MG, Gleeson JP, O'Brien FJ. Mesenchymal stem cell fate is regulated by the composition and mechanical properties of collagenglycosaminoglycan scaffolds. J Mech Behav Biomed Mater. 2012; 11:53-62.

45. Haugh MG, Murphy CM, McKiernan RC, Altenbuchner C, O'Brien FJ. Crosslinking and Mechanical Properties Significantly Influence Cell Attachment, Proliferation, and Migration Within Collagen Glycosaminoglycan Scaffolds. Tissue Eng Part A. 2011; 17(9-10):1201-8.

46. Freyman TM, Yannas IV, Pek YS, Yokoo R, Gibson LJ. Micromechanics of fibroblast contraction of a collagen-GAG matrix. Exp Cell Res. 2001; 269(1):140-53. 47. Jungreuthmayer C, Jaasma MJ, Al-Munajjed AA, Zanghellini J, Kelly DJ, O'Brien FJ. Deformation simulation of cells seeded on a collagen-GAG scaffold in a flow perfusion bioreactor using a sequential 3D CFD-elastostatics model. Med Eng Phys. 2009; 31(4):420-7. 
48. Corin KA, Gibson LJ. Cell contraction forces in scaffolds with varying pore size and cell density. Biomaterials. 2010; 31(18):4835-45.

49. Gibson LJ, Ashby MF. Cellular solids: structures \& properties. Second Edition ed. Oxford: Pergamon Press; 1997. 510 p.

50. Her GJ, Wu HC, Chen MH, Chen MY, Chang SC, Wang TW. Control of threedimensional substrate stiffness to manipulate mesenchymal stem cell fate toward neuronal or glial lineages. Acta Biomater. 2013; 9(2):5170-80.

51. Wingate K, Bonani W, Tan Y, Bryant SJ, Tan W. Compressive elasticity of three-dimensional nanofiber matrix directs mesenchymal stem cell differentiation to vascular cells with endothelial or smooth muscle cell markers. Acta Biomater. 2012; 8(4):1440-9.

52. Romano NH, Sengupta D, Chung C, Heilshorn SC. Protein-engineered biomaterials: nanoscale mimics of the extracellular matrix. Biochim Biophys Acta. $2011 ; 1810(3): 339-49$.

53. Trappmann B, Chen CS. How cells sense extracellular matrix stiffness: a material's perspective. Curr Opin Biotechnol. 2013; 24(5):948-53.

54. Davidenko N, Schuster CF, Bax DV, Raynal N, Farndale RW, Best SM, et al. Control of crosslinking for tailoring collagen-based scaffolds stability and mechanics. Acta Biomater. 2015.

55. Charati MB, Ifkovits JL, Burdick JA, Linhardt JG, Kiick KL. Hydrophilic elastomeric biomaterials based on resilin-like polypeptides. Soft matter. 2009; 5(18):3412-6.

56. Straley KS, Heilshorn SC. Dynamic, 3D-Pattern Formation Within EnzymeResponsive Hydrogels. Adv Mater. 2009; 21(41):4148-52.

57. Houseman BT, Mrksich M. The microenvironment of immobilized Arg-GlyAsp peptides is an important determinant of cell adhesion. Biomaterials. 2001; 22(9):943-55.

58. Desai RM, Koshy ST, Hilderbrand SA, Mooney DJ, Joshi NS. Versatile click alginate hydrogels crosslinked via tetrazine-norbornene chemistry. Biomaterials. 2015; 50:30-7.

59. DeForest CA, Anseth KS. Cytocompatible click-based hydrogels with dynamically tunable properties through orthogonal photoconjugation and photocleavage reactions. Nat Chem. 2011; 3(12):925-31.

60. Nimmo CM, Shoichet MS. Regenerative Biomaterials that "Click": Simple, Aqueous-Based Protocols for Hydrogel Synthesis, Surface Immobilization, and 3D Patterning. Bioconjug Chem. 2011; 22(11):2199-209.

61. Kakinoki S, Seo JH, Inoue Y, Ishihara K, Yui N, Yamaoka T. Mobility of the Arg-Gly-Asp ligand on the outermost surface of biomaterials suppresses integrinmediated mechanotransduction and subsequent cell functions. Acta Biomater. 2015; $13: 42-51$.

62. Kloxin AM, Kasko AM, Salinas CN, Anseth KS. Photodegradable hydrogels for dynamic tuning of physical and chemical properties. Science. 2009; 324(5923):5963.

63. Petersen S, Alonso JM, Specht A, Duodu P, Goeldner M, del Campo A. Phototriggering of cell adhesion by caged cyclic RGD peptides. Angew Chem Int Ed Engl. 2008; 47(17):3192-5. 
64. Wylie RG, Ahsan S, Aizawa Y, Maxwell KL, Morshead CM, Shoichet MS. Spatially controlled simultaneous patterning of multiple growth factors in threedimensional hydrogels. Nat Mater. 2011; 10(10):799-806.

65. Miron-Mendoza M, Koppaka V, Zhou C, Petroll WM. Techniques for assessing $3-\mathrm{D}$ cell-matrix mechanical interactions in vitro and in vivo. Exp Cell Res. 2013; 319(16):2470-80.

66. Brackmann C, Esguerra M, Olausson D, Delbro D, Krettek A, Gatenholm P, et al. Coherent anti-Stokes Raman scattering microscopy of human smooth muscle cells in bioengineered tissue scaffolds. J Biomed Opt. 2011; 16(2):021115.

67. Rice WL, Kaplan DL, Georgakoudi I. Two-photon microscopy for noninvasive, quantitative monitoring of stem cell differentiation. PLoS ONE. 2010; 5(4):e10075.

68. Gudur MS, Rao RR, Peterson AW, Caldwell DJ, Stegemann JP, Deng CX. Noninvasive quantification of in vitro osteoblastic differentiation in $3 \mathrm{D}$ engineered tissue constructs using spectral ultrasound imaging. PLoS ONE. 2014; 9(1):e85749. 69. Vielreicher M, Schurmann S, Detsch R, Schmidt MA, Buttgereit A, Boccaccini A, et al. Taking a deep look: modern microscopy technologies to optimize the design and functionality of biocompatible scaffolds for tissue engineering in regenerative medicine. J R Soc Interface. 2013; 10(86):20130263.

70. Schultz KM, Anseth KS. Monitoring degradation of matrix metalloproteinasescleavable PEG hydrogels via multiple particle tracking microrheology. Soft matter. 2013; 9(5):1570-9.

71. Ranga A, Lutolf MP. High-throughput approaches for the analysis of extrinsic regulators of stem cell fate. Curr Opin Cell Biol. 2012; 24(2):236-44.

72. Gobaa S, Hoehnel S, Roccio M, Negro A, Kobel S, Lutolf MP. Artificial niche microarrays for probing single stem cell fate in high throughput. Nat Methods. 2011; 8(11):949-55.

73. Huebsch N, Lippens E, Lee K, Mehta M, Koshy ST, Darnell MC, et al. Matrix elasticity of void-forming hydrogels controls transplanted-stem-cell-mediated bone formation. Nat Mater. 2015; 14(12):1269-77.

74. Beer FP, Johnston ER, DeWolf JT. Mechanics of materials. 4th ed. Boston: McGraw-Hill Higher Education; 2006. 
A

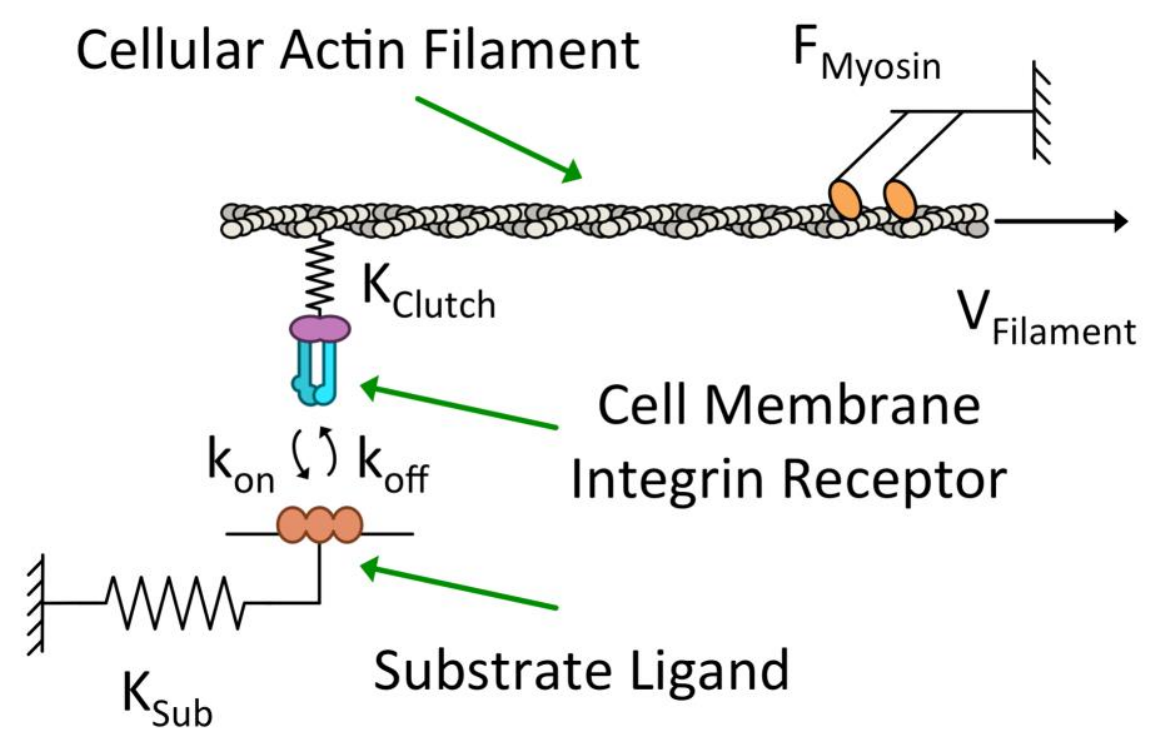

B

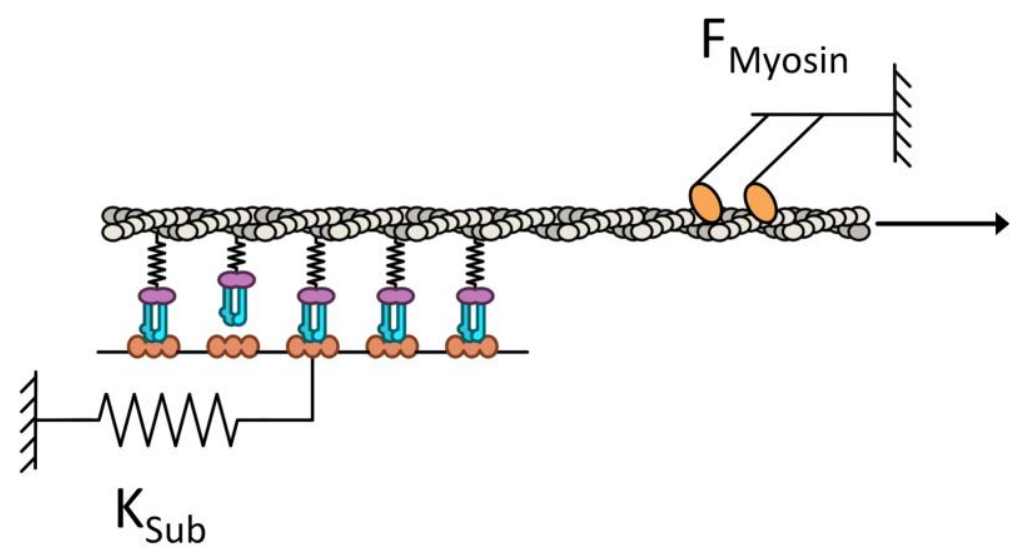

Figure 1. The molecular clutch model of integrin-ligand interactions. (A) Forces applied by myosin motors (FMyosin) result in the retrograde flow of actin filaments (VFilament). This flow is resisted by the formation of bonds, termed molecular clutches, between actin filaments and the substrate. The formation of these bonds is initiated by the stochastic binding and unbinding, at rates $\mathrm{k}_{\mathrm{on}}$ and $\mathrm{k}_{\text {off }}$ respectively, of integrin receptors present at the cell membrane to ligands presented on the substrate surface. This enables the substrate bound integrins to bind with actin filaments resulting in a connection between the substrate and actin filaments. Subsequent rearward motion of actin leads to the deformation of both the clutches and the substrate, with the tension 
developed in the filament proportional to the stiffness of both the clutches ( $\left.\mathrm{K}_{\mathrm{Clutch}}\right)$ and the substrate (KSub). (B) As several molecular clutches can bind to a single actin filament, the mechanical resistance sensed by a cell is defined by the number of potential clutches, the clutch stiffness $\left(K_{\text {Clutch }}\right)$ and the clutch binding rates in addition to the substrate stiffness $\left(\mathrm{K}_{\mathrm{Sub}}\right)$. For a given cell type, the density and identity of the ligands presented by the substrate governs the clutch characteristics and are therefore important material parameters in studies of cell-substrate mechanotransduction. Schematic adapted from references $(22,42)$. 

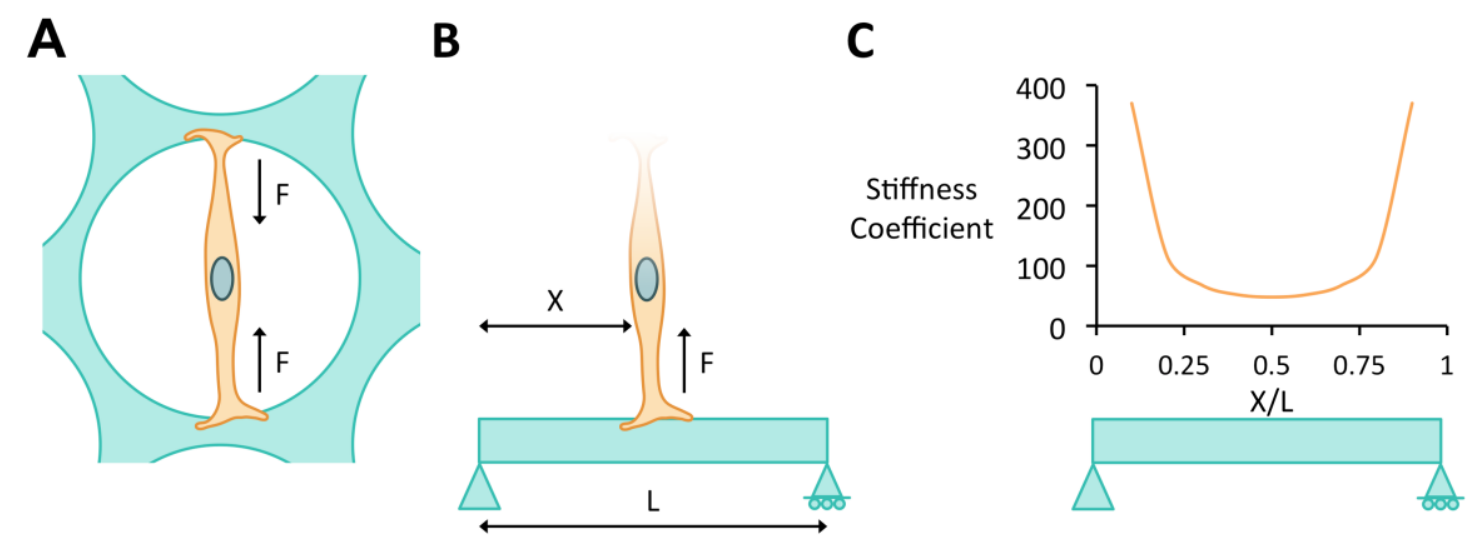

Figure 2. Mechanical heterogeneity within 3D macro-porous substrates. (A) Cells can attach across pores generating tensile forces that have been observed to buckle struts within macro-porous substrates $(46,48)$. (B) The assumption can be made that each strut within a macro-porous substrate behaves as a simply supported beam of length $\mathrm{L}$ and that the tensile force generated by the cell acts as a point load (F) applied at a location $\mathrm{X}$ along the beam length. (C) The application of beam mechanics theory provides an approximation of the stiffness coefficient $\left(\mathrm{C}\right.$, where $\mathrm{KSub}=\mathrm{C} * \mathrm{EI} / \mathrm{L}^{3}, \mathrm{E}$ is Young's modulus of the strut material and I is the second moment of inertia of the strut geometry), demonstrating that the stiffness increases as the point of cellular attachment moves away from the strut mid-point (74). This is a simplified model of cellular attachment, intended solely to illustrate the heterogeneity within macro-porous substrates. Due to the wide range of possible cellular attachment orientations and pore geometries, computational analysis is required to fully characterize the mechanical environment sensed by cells within such substrates. 


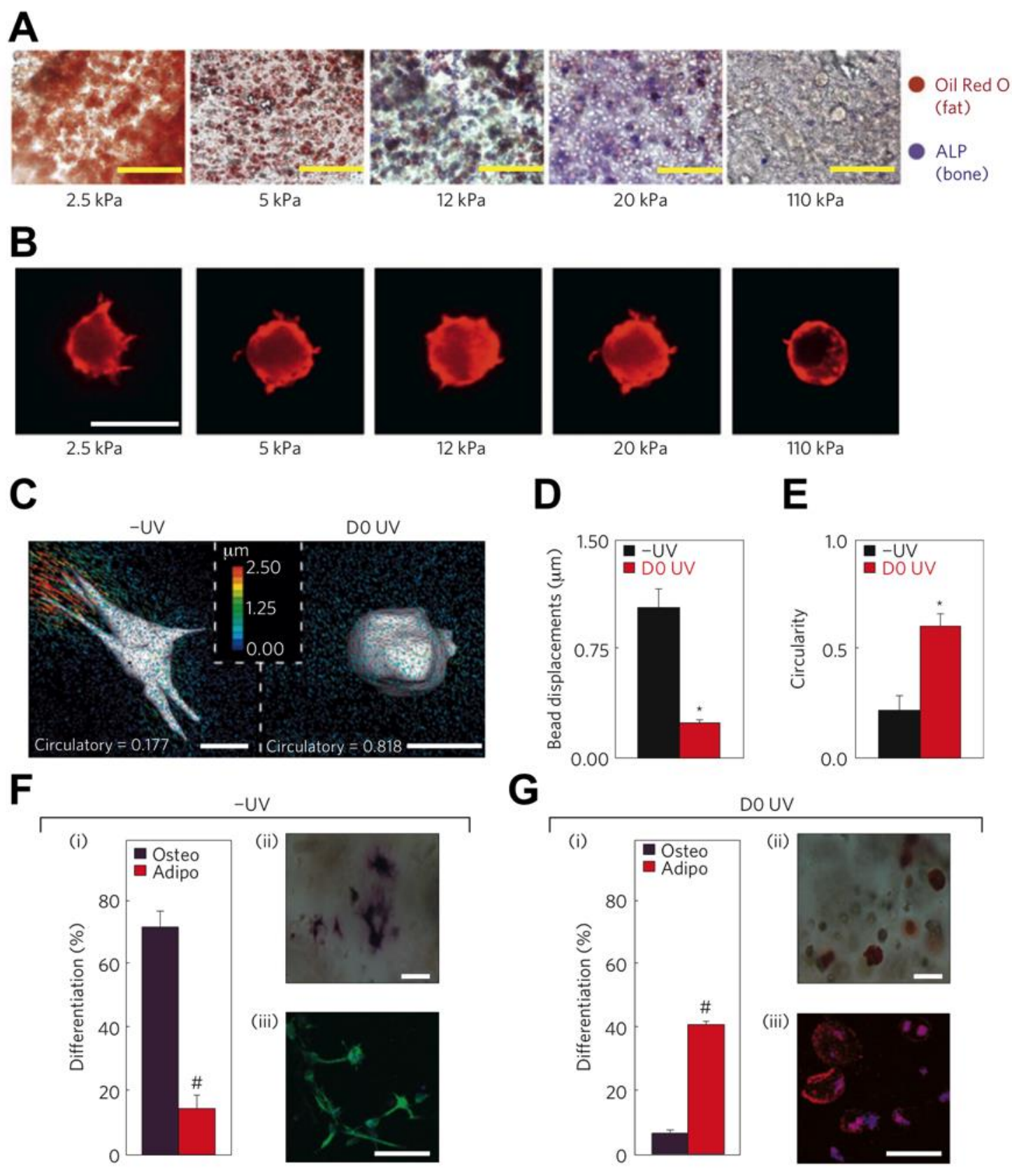

Figure 3. The fate of MSCs encapsulated within 3D non-macro-porous hydrogel substrates. (A) Histological assessment of MSC fate reveals a biphasic response to stiffness after 1 week of culture within physically-crosslinked, alginate hydrogels with moduli ranging from 2.5-110 $\mathrm{kPa}$. Scale bars: $100 \mu \mathrm{m}$. (B) Phalloidin staining of actin within physically-crosslinked alginate hydrogels reveals no correlation between cell shape $2 \mathrm{~h}$ after encapsulation. Scale bar: $10 \mu \mathrm{m}$. A-B reproduced with permission (11). 
Copyright 2010, Nature Publishing Group. (C-E) 3D traction force microscopy (TFM) reveals increased cell spreading and bead displacement within degradable (-UV) compared to non-degradable (D0 UV) MeHA hydrogels. Bead displacement is a measure of hydrogel deformation as a result of both cell traction and substrate degradation. Scale bars: $15 \mu \mathrm{m}$. (F-G) Quantification of histological staining for differentiation markers after 14 days of culture in mixed induction media demonstrates that MeHA hydrogel degradability correlated with increased osteogenic differentiation, whereas significant adipogenesis was observed in non-degradable hydrogels. Osteogenic differentiation was assed by staining for alkaline phosphatase (Fii) and osteocalcin (Fiii, Green). Adipogenic differentiation was assed by staining for lipid droplets (Oil Red O, Gii) and fatty acid binding protein (Giii, Red). Scale bars: $25 \mu \mathrm{m}$ (Fii, Gii) and $20 \mu \mathrm{m}$ (Fiii, Giii). C-G reproduced with permission (12). Copyright 2013, Nature Publishing Group. 